\title{
On Equivalence of Infinitary Formulas under the Stable Model Semantics
}

\author{
Amelia Harrison, Vladimir Lifschitz \\ University of Texas \\ Miroslaw Truszczynski \\ University of Kentucky \\ submitted 4 December 2013; revised 21 February 2014; accepted 10 March 2014
}

\begin{abstract}
Propositional formulas that are equivalent in intuitionistic logic, or in its extension known as the logic of here-and-there, have the same stable models. We extend this theorem to propositional formulas with infinitely long conjunctions and disjunctions and show how to apply this generalization to proving properties of aggregates in answer set programming.
\end{abstract}

KEYWORDS: answer set programming, semantics of aggregates, intuitionistic logic, strong equivalence

\section{Introduction}

This note is about the extension of the stable model semantics to infinitary propositional formulas defined by Truszczynski (2012). That extension, introduced originally as a tool for proving a theorem about the logic FO(ID), has been used also to prove a new generalization of Fages' theorem (Lifschitz and Yang 2012).

One of the reasons why stable models of infinitary formulas are important is that they are closely related to aggregates in answer set programming (ASP). The semantics of aggregates proposed by Ferraris (2005, Section 4.1) treats a ground aggregate as shorthand for a propositional formula. An aggregate with variables has to be grounded before that semantics can be applied to it. For instance, to explain the precise meaning of the expression $1\{p(X)\}$ ("there exists at least one object with the property $p ")$ in the body of an ASP rule we first rewrite it as

$$
1\left\{p\left(t_{1}\right), \ldots, p\left(t_{n}\right)\right\}
$$

where $t_{1}, \ldots, t_{n}$ are all ground terms in the language of the program, and then turn it into the propositional formula

$$
p\left(t_{1}\right) \vee \cdots \vee p\left(t_{n}\right) .
$$

But this description of the meaning of $1\{p(X)\}$ implicitly assumes that the Herbrand universe of the program is finite. If the program contains function symbols then an infinite disjunction has to be used instead of (1). 
There is nothing exotic or noncomputable about ASP programs containing both aggregates and function symbols. For instance, the program

$$
\begin{aligned}
& p(f(a)) \\
& q \leftarrow 1\{p(X)\}
\end{aligned}
$$

has simple intuitive meaning, and its stable model $\{p(f(a)), q\}$ can be computed by Version 3 of the answer set solver CLINGo. ${ }^{1}$ More generally, stable models of infinitary propositional formulas in the sense of Truszczynski (2012) can be used to define the semantics of aggregates in the input language of CLINGO (Harrison 2013); this is our main motivation for studying their properties.

\section{Remark 1}

Attempts to define the semantics of aggregates for other ASP languages encounter similar difficulties if the Herbrand universe is infinite. For instance, the definition of a ground instance of a rule in Section 2.2 of the ASP Core document (https://www.mat.unical.it /aspcomp2013/files/ASP-CORE-2.0.pdf, Version 2.02) talks about replacing the expression $\left\{e_{1} ; \ldots ; e_{n}\right\}$ in a rule with a set denoted by inst $\left(\left\{e_{1} ; \ldots ; e_{n}\right\}\right)$. But that set can be infinite and then it cannot be included in a rule.

Our goal here is to develop methods for proving that pairs $F, G$ of infinitary formulas have the same stable models. From the results of Pearce (1997) and Ferraris (2005) we know that in the case of grounded logic programs in the sense of Gelfond and Lifschitz (1988) and, more generally, sets of finite propositional formulas, it is sufficient to check that the equivalence $F \leftrightarrow G$ is provable intuitionistically. Some extensions of intuitionistic propositional logic, including the logic of here-and-there, can be used as well. In this note, we extend these results to deductive systems of infinitary propositional logic.

This goal is closely related to the idea of strong equivalence (Lifschitz, Pearce, Valverde, 2001). The provability of $F \leftrightarrow G$ in the deductive systems of infinitary logic described below guarantees not only that $F$ and $G$ have the same stable models, but also that for any set $\mathcal{H}$ of infinitary formulas, $\mathcal{H} \cup\{F\}$ and $\mathcal{H} \cup\{G\}$ have the same stable models.

We review the stable model semantics of infinitary propositional formulas in Section 2. Then we define a basic infinitary system of natural deduction, similar to propositional intuitionistic logic (Section 3), and study its properties (Section 4). The main theorem is stated and proved in Section 5, and applied to examples involving aggregates in Section 6. A useful extension of the basic system is discussed in Section 7.

A preliminary report on this work was presented at the 2013 International Conference on Logic Programming and Nonmonotonic Reasoning (Harrison, Lifschitz, Truszczynski, 2013).

\footnotetext{
${ }^{1}$ http://potassco. sourceforge.net.
} 


\section{Stable Models of Infinitary Propositional Formulas}

The definitions of infinitary formulas and their stable models given below are equivalent to the definitions proposed by Truszczynski (2012).

Let $\sigma$ be a propositional signature, that is, a set of propositional atoms. The sets $\mathcal{F}_{0}^{\sigma}, \mathcal{F}_{1}^{\sigma}, \ldots$ are defined as follows:

- $\mathcal{F}_{0}^{\sigma}=\sigma$,

- $\mathcal{F}_{i+1}^{\sigma}$ is obtained from $\mathcal{F}_{i}^{\sigma}$ by adding expressions $\mathcal{H}^{\vee}$ and $\mathcal{H}^{\wedge}$ for all subsets $\mathcal{H}$ of $\mathcal{F}_{i}^{\sigma}$, and expressions $F \rightarrow G$ for all $F, G \in \mathcal{F}_{i}^{\sigma}$.

The elements of $\bigcup_{i=0}^{\infty} \mathcal{F}_{i}^{\sigma}$ are called (infinitary) formulas over $\sigma$.

Remark 2

This definition differs from the syntax introduced in early work on infinitary propositional formulas (Scott and Tarski 1958; Karp 1964) in several ways. It treats the collection $\mathcal{H}$ of conjunctive or disjunctive terms as a set, rather than a family indexed by ordinals. Thus there is no order among conjunctive or disjunctive terms in this framework, and there can be no repetitions among them. More importantly, there is no restriction here on the cardinality of the set of conjunctive or disjunctive terms. On the other hand, in the hierarchy $\mathcal{F}_{i}^{\sigma}$ of sets of formulas, $i$ is a natural number; transfinite levels are not allowed.

A set $\mathcal{H}$ of formulas is bounded if it is contained in one of the sets $\mathcal{F}_{i}^{\sigma}$. For a bounded set $\mathcal{H}$ of formulas, $\mathcal{H}^{\wedge}$ and $\mathcal{H}^{\vee}$ are infinitary formulas.

The symbol $\perp$ will be understood as an abbreviation for $\emptyset^{\vee} ; \neg F$ stands for $F \rightarrow \perp$, and $F \leftrightarrow G$ stands for $(F \rightarrow G) \wedge(G \rightarrow F)$.

We will write $\{F, G\}^{\wedge}$ as $F \wedge G$, and $\{F, G\}^{\vee}$ as $F \vee G$. This convention allows us to view finite propositional formulas over $\sigma$ as a special case of infinitary formulas. For any bounded family $\left\{F_{\alpha}\right\}_{\alpha \in A}$ of formulas, we denote the formula $\left\{F_{\alpha}: \alpha \in A\right\}^{\wedge}$ by $\bigwedge_{\alpha \in A} F_{\alpha}$, and similarly for disjunctions.

Subsets of a signature $\sigma$ will be also called its interpretations. The satisfaction relation between an interpretation $I$ and a formula $F$ is defined as follows:

- For every $p \in \sigma, I \models p$ if $p \in I$.

- $I \models \mathcal{H}^{\wedge}$ if for every formula $F \in \mathcal{H}, I \models F$.

- $I \models \mathcal{H}^{\vee}$ if there is a formula $F \in \mathcal{H}$ such that $I \models F$.

- $I \models F \rightarrow G$ if $I \not \models F$ or $I \models G$.

We say that $I$ satisfies a set $\mathcal{H}$ of formulas if $I$ satisfies all elements of $\mathcal{H}$. Two sets of formulas are equivalent to each other if they are satisfied by the same interpretations. A formula $F$ is tautological if it is satisfied by all interpretations.

The reduct $F^{I}$ of a formula $F$ with respect to an interpretation $I$ is defined as follows:

- For $p \in \sigma, p^{I}=\perp$ if $I \not \forall p$; otherwise $p^{I}=p$.

- $\left(\mathcal{H}^{\wedge}\right)^{I}=\left\{G^{I} \mid G \in \mathcal{H}\right\}^{\wedge}$.

- $\left(\mathcal{H}^{\vee}\right)^{I}=\left\{G^{I} \mid G \in \mathcal{H}\right\}^{\vee}$.

- $(G \rightarrow H)^{I}=\perp$ if $I \not \forall G \rightarrow H$; otherwise $(G \rightarrow H)^{I}=G^{I} \rightarrow H^{I}$. 
The reduct $\mathcal{H}^{I}$ of a set $\mathcal{H}$ of formulas is the set consisting of the reducts of the elements of $\mathcal{H}$. An interpretation $I$ is a stable model of a set $\mathcal{H}$ of formulas if it is minimal w.r.t. set inclusion among the interpretations satisfying $\mathcal{H}^{I}$; a stable model of a formula $F$ is a stable model of singleton $\{F\}$. This is a straightforward extension of the definition of a stable model due to Ferraris (2005) to infinitary formulas.

It is easy to see that $I \models F^{I}$ iff $I \models F$. It follows that every stable model of $\mathcal{H}$ satisfies $\mathcal{H}$.

\section{Basic Infinitary System of Natural Deduction}

Inference rules of the deductive system described below are similar to the standard natural deduction rules of propositional logic. ${ }^{2}$ Its derivable objects are (infinitary) sequents - expressions of the form $\Gamma \Rightarrow F$, where $F$ is an infinitary formula, and $\Gamma$ is a finite set of infinitary formulas (" $F$ under assumptions $\Gamma$ "). To simplify notation, we will write $\Gamma$ as a list. We will identify a sequent of the form $\Rightarrow F$ with the formula $F$.

There is one axiom schema $F \Rightarrow F$. The inference rules are the introduction and elimination rules for the propositional connectives

$$
\begin{array}{ll}
(\wedge I) \frac{\Gamma \Rightarrow H \quad \text { for all } H \in \mathcal{H}}{\Gamma \Rightarrow \mathcal{H}^{\wedge}} & (\wedge E) \frac{\Gamma \Rightarrow \mathcal{H}^{\wedge}}{\Gamma \Rightarrow H} \quad(H \in \mathcal{H}) \\
(\vee I) \frac{\Gamma \Rightarrow H}{\Gamma \Rightarrow \mathcal{H}^{\vee}} \quad(H \in \mathcal{H}) & (\vee E) \frac{\Gamma \Rightarrow \mathcal{H}^{\vee}}{\Delta, H \Rightarrow F \quad \text { for all } H \in \mathcal{H}} \\
(\rightarrow I) \frac{\Gamma, F \Rightarrow G}{\Gamma \Rightarrow F \rightarrow G} & (\rightarrow E) \frac{\Gamma \Rightarrow F \quad \Delta \Rightarrow F \rightarrow G}{\Gamma, \Delta \Rightarrow G},
\end{array}
$$

where $\mathcal{H}$ is a bounded set of formulas, and the weakening rule

$$
(W) \frac{\Gamma \Rightarrow F}{\Gamma, \Delta \Rightarrow F}
$$

Remark 3

The usual conjunction introduction rule is

$$
\frac{\Gamma \Rightarrow F \quad \Delta \Rightarrow G}{\Gamma, \Delta \Rightarrow F \wedge G}
$$

the corresponding infinitary rule above is similar to the more restrictive version:

$$
\frac{\Gamma \Rightarrow F \quad \Gamma \Rightarrow G}{\Gamma \Rightarrow F \wedge G}
$$

In the presence of the weakening rule $(W)$, the two versions are equivalent to each other. The situation with disjunction elimination is similar. The usual contradiction rule

$$
\text { (C) } \frac{\Gamma \Rightarrow \perp}{\Gamma \Rightarrow F}
$$

\footnotetext{
${ }^{2}$ See, for instance, (Lifschitz et al. 2008, Section 1.2.1).
} 
is a special case of $(\vee E)$. We do not include the law of the excluded middle in the set of axioms, so that this deductive system is similar to intuitionistic, rather than classical, propositional logic.

The set of theorems of the basic system is the smallest set of sequents that includes the axioms of the system and is closed under the application of its inference rules. We say that formulas $F$ and $G$ are equivalent in the basic system if $F \leftrightarrow G$ is a theorem of the basic system. The reason why we are interested in this relation is that formulas equivalent in the basic system have the same stable models, as discussed in Section 5 below.

Example 1

Let $\left\{F_{i}\right\}_{i \in \mathbb{N}}$ be a bounded family of formulas. We will check that the formula

$$
F_{0} \wedge \bigwedge_{i \geq 0}\left(F_{i} \rightarrow F_{i+1}\right)
$$

is equivalent in the basic system to the formula $\bigwedge_{i \geq 0} F_{i}$. The sequent

$$
F_{0} \wedge \bigwedge_{i \geq 0}\left(F_{i} \rightarrow F_{i+1}\right) \Rightarrow F_{0} \wedge \bigwedge_{i \geq 0}\left(F_{i} \rightarrow F_{i+1}\right)
$$

belongs to the set of theorems of the basic system. Consequently so do the sequents

$$
F_{0} \wedge \bigwedge_{i \geq 0}\left(F_{i} \rightarrow F_{i+1}\right) \Rightarrow F_{0}
$$

and

$$
F_{0} \wedge \bigwedge_{i \geq 0}\left(F_{i} \rightarrow F_{i+1}\right) \Rightarrow F_{j} \rightarrow F_{j+1}
$$

for all $j \geq 0$. Consequently the sequents

$$
F_{0} \wedge \bigwedge_{i \geq 0}\left(F_{i} \rightarrow F_{i+1}\right) \Rightarrow F_{j}
$$

for all $j \geq 0$ belong to the set of theorems as well (by induction on $j$ ). Consequently so does the sequent

$$
F_{0} \wedge \bigwedge_{i \geq 0}\left(F_{i} \rightarrow F_{i+1}\right) \Rightarrow \bigwedge_{i \geq 0} F_{i}
$$

A similar argument (except that induction is not needed) shows that the sequent

$$
\bigwedge_{i \geq 0} F_{i} \Rightarrow F_{0} \wedge \bigwedge_{i \geq 0}\left(F_{i} \rightarrow F_{i+1}\right)
$$

is a theorem of the basic system also. Consequently so is the sequent

$$
\Rightarrow F_{0} \wedge \bigwedge_{i \geq 0}\left(F_{i} \rightarrow F_{i+1}\right) \leftrightarrow \bigwedge_{i \geq 0} F_{i} .
$$

This argument could be expressed more concisely, without explicit references to the set of theorems of the basic system, as follows. Assume (2). Then $F_{0}$ and, for every $i \geq 0, F_{i} \rightarrow F_{i+1}$. Then, by induction, $F_{i}$ for every $i$. And so forth. This style of presentation is used in the next example. 
Example 2

Let $\left\{F_{\alpha}\right\}_{\alpha \in A}$ be a bounded family of formulas, and let $G$ be a formula. Let us show that

$$
\left(\bigvee_{\alpha \in A} F_{\alpha}\right) \rightarrow G
$$

is equivalent in the basic system to the formula

$$
\bigwedge_{\alpha \in A}\left(F_{\alpha} \rightarrow G\right)
$$

Left-to-right: assume (3) and $F_{\alpha}$. Then $\bigvee_{\alpha \in A} F_{\alpha}$, and consequently $G$. Thus we established $F_{\alpha} \rightarrow G$ under assumption (3) alone for every $\alpha$, and consequently established (4) under this assumption as well. Right-to-left: assume (4) and $\bigvee_{\alpha \in A} F_{\alpha}$, and consider the cases corresponding to the disjunctive terms of this disjunction. Assume $F_{\alpha}$. From (4), $F_{\alpha} \rightarrow G$, and consequently $G$. Thus we established $G$ in each case, so that (3) follows from (4) alone.

It is easy to see that the infinitary counterparts of the intuitionistically provable De Morgan's laws

$$
\bigvee_{F \in \mathcal{H}} \neg F \rightarrow \neg \bigwedge_{F \in \mathcal{H}} F
$$

and

$$
\bigwedge_{F \in \mathcal{H}} \neg F \leftrightarrow \neg \bigvee_{F \in \mathcal{H}} F,
$$

where $\mathcal{H}$ is a bounded set of formulas, are theorems of the basic system. So are the infinitary distributivity laws

$$
\left(\bigvee_{\left\{F_{i}\right\}_{i \in I}} \bigwedge_{i \in I} F_{i}\right) \rightarrow\left(\bigwedge_{i \in I} \bigvee_{F \in \mathcal{H}_{i}} F\right)
$$

and

$$
\left(\bigvee_{i \in I} \bigwedge_{F \in \mathcal{H}_{i}} F\right) \rightarrow\left(\bigwedge_{\left\{F_{i}\right\}_{i \in I}} \bigvee_{i \in I} F_{i}\right)
$$

for every non-empty family $\left\{\mathcal{H}_{i}\right\}_{i \in I}$ of sets of formulas such that its union is bounded. The disjunction in the antecedent of (7) and the conjunction in the consequent of (8) extend over all elements $\left\{F_{i}\right\}_{i \in I}$ of the Cartesian product of the family $\left\{\mathcal{H}_{i}\right\}_{i \in I}$. In Section 7 we discuss an extension of the basic system in which we postulate the converses of implications (5), (7), and (8).

\section{Properties of the Basic System}

The following property of the basic system is easy to verify. 
Proposition 1

If a sequent consisting of finite formulas is intuitionistically provable then it is a theorem of the basic system.

Recall that we define the set of theorems of the basic system to be the smallest set of formulas that includes the axioms and is closed under the inference rules. When we want to prove that every theorem of the basic system has a certain property $P$, it is clearly sufficient to check that every axiom has the property $P$, and that the set of sequents that have the property $P$ is closed under the application of the inference rules. In this way we can establish, in particular, the following fact:

Proposition 2

For any theorem $\Gamma \Rightarrow F$ of the basic system, the formula $\Gamma^{\wedge} \rightarrow F$ is tautological.

Remark 4

The assertion of Proposition 2 will remain true even if we extend the set of axioms to include the law of the excluded middle

$$
F \vee \neg F \text {. }
$$

The converse is not true, however, even in the presence of this axiom schema. This fact can be established by standard methods used to prove incompleteness in infinitary logic, which utilize the Downward Löwenheim-Skolem Theorem and the Mostowski Collapsing Lemma. ${ }^{3}$ We can make the system complete by postulating the following infinitary version of the law of the excluded middle:

$$
\bigvee_{J \subseteq I}\left(\bigwedge_{j \in J} F_{j} \wedge \bigwedge_{j \in I \backslash J} \neg F_{j}\right),
$$

for any non-empty bounded family $\left\{F_{i}\right\}_{i \in I}$ of formulas. ${ }^{4}$

Let $\sigma$ and $\sigma^{\prime}$ be disjoint signatures. In this section, a substitution is a bounded family of formulas over $\sigma$ with index set $\sigma^{\prime}$. For any substitution $\phi$ and any formula $F$ over the signature $\sigma \cup \sigma^{\prime}, \phi F$ stands for the formula over $\sigma$ formed as follows:

- If $F \in \sigma$ then $\phi F=F$.

- If $F \in \sigma^{\prime}$ then $\phi F=\phi_{F}$.

- If $F$ is $\mathcal{H}^{\wedge}$ then $\phi F=\{\phi G \mid G \in \mathcal{H}\}^{\wedge}$.

- If $F$ is $\mathcal{H}^{\vee}$ then $\phi F=\{\phi G \mid G \in \mathcal{H}\}^{\vee}$.

- If $F$ is $G \rightarrow H$ then $\phi F=\phi G \rightarrow \phi H$.

Formulas of the form $\phi F$ will be called instances of $F$.

\footnotetext{
3 John Schlipf, personal communication.

4 The proof of this fact is similar to the proof of completeness of classical propositional logic due to Kalmár (1935). For any interpretation $I$, let $L_{I}$ denote the conjunction of the corresponding set of literals. It is easy to check by induction that for any formula $F, L_{I} \rightarrow F$ is a theorem of the basic system if $I$ satisfies $F$, and $L_{I} \rightarrow \neg F$ is a theorem of the basic system otherwise. The completeness of the basic system with (10) added as an axiom schema easily follows.
} 
Proposition 3

If $F$ is a theorem of the basic system then every instance of $F$ is a theorem of the basic system also.

Proof

The notation $\phi F$ extends to sequents in a natural way. The property " $\phi S$ is a theorem of the basic system" holds for every axiom $S$ of the basic system, and it is preserved by all inference rules.

We will refer to Proposition 3 as the substitution property of the basic system.

Example 3

We will show that for any formulas $F$, $G$, the formula $\neg(F \vee G)$ is equivalent to $\neg F \wedge \neg G$ in the basic system. Note first that the formula

$$
\neg(p \vee q) \leftrightarrow \neg p \wedge \neg q
$$

is intuitionistically provable. By Proposition 1, it follows that it is a theorem of the basic system. The equivalence

$$
\neg(F \vee G) \leftrightarrow \neg F \wedge \neg G
$$

is an instance of (11): take $\phi_{p}=F, \phi_{q}=G$. By the substitution property, it follows that it is a theorem of the basic system as well.

Proposition 4

For any substitutions $\phi, \psi$ with the same index set, the implication

$$
\bigwedge_{p}\left(\phi_{p} \leftrightarrow \psi_{p}\right) \rightarrow(\phi F \leftrightarrow \psi F)
$$

(where $p$ ranges over the indices) is a theorem of the basic system.

Proof

The proof is by induction on $j$ such that $F \in \mathcal{F}_{j}^{\sigma \cup \sigma^{\prime}}$, and it considers several cases, depending on the syntactic form of $F$. Assume, for instance, that $F$ is $\mathcal{H}^{\vee}$. Then

$$
\phi F=\{\phi G \mid G \in \mathcal{H}\}^{\vee}, \quad \psi F=\{\psi G \mid G \in \mathcal{H}\}^{\vee} .
$$

By the induction hypothesis, for each $G$ in $\mathcal{H}$, the implication

$$
\bigwedge_{p}\left(\phi_{p} \leftrightarrow \psi_{p}\right) \rightarrow(\phi G \leftrightarrow \psi G)
$$

is a theorem of the basic system. We need to show that

$$
\bigwedge_{p}\left(\phi_{p} \leftrightarrow \psi_{p}\right) \rightarrow\left(\{\phi G \mid G \in \mathcal{H}\}^{\vee} \leftrightarrow\{\psi G \mid G \in \mathcal{H}\}^{\vee}\right)
$$

is a theorem of the basic system also. Assume

$$
\bigwedge_{p}\left(\phi_{p} \leftrightarrow \psi_{p}\right)
$$

and $\{\phi G \mid G \in \mathcal{H}\}^{\vee}$, and consider the cases corresponding to the terms of this disjunction. Assume $\phi G$. Then, by (12) and (14), $\psi G$. We can conclude $\{\psi G \mid G \in \mathcal{H}\}^{\vee}$, that is, $\psi F$. So we established the implication $\phi F \rightarrow \psi F$. The implication in the other direction is proved in a similar way. 
Corollary 1

If for every index $p, \phi_{p}$ is equivalent to $\psi_{p}$ in the basic system then $\phi F$ is equivalent to $\psi F$ in the basic system.

We will refer to this corollary as the replacement property of the basic system.

Example 4

The formula

$$
\bigwedge_{k \geq 1}\left(p_{k} \rightarrow \neg p_{k}\right) \rightarrow p_{0}
$$

is equivalent to

$$
\bigwedge_{k \geq 1} \neg p_{k} \rightarrow p_{0}
$$

in the basic system, because (16) can be obtained from (15) by replacing $p_{k} \rightarrow \neg p_{k}$ with the intuitionistically equivalent $\neg p_{k}$. More formally, let $q_{k}(k \geq 1)$ be the indices and let $F$ be $\bigwedge_{k \geq 1} q_{k} \rightarrow p_{0}$. For the substitutions

$$
\phi_{q_{k}}=p_{k} \rightarrow \neg p_{k}, \quad \psi_{q_{k}}=\neg p_{k},
$$

$\phi F$ is (15), and $\psi F$ is (16). By the replacement property, (15) is equivalent to (16).

\section{Relation of the Basic System to Stable Models}

Main Theorem

For any set $\mathcal{H}$ of formulas,

(a) if a formula $F$ is a theorem of the basic system then $\mathcal{H} \cup\{F\}$ has the same stable models as $\mathcal{H}$;

(b) if $F$ is equivalent to $G$ in the basic system then $\mathcal{H} \cup\{F\}$ and $\mathcal{H} \cup\{G\}$ have the same stable models.

\section{Lemma 1}

For any formula $F$ and interpretation $I$, if $I$ does not satisfy $F$ then $F^{I} \Rightarrow \perp$ is a theorem of the basic system.

The proof is straightforward by induction on $i$ such that $F \in \mathcal{F}_{i}^{\sigma}$.

By $\Gamma^{I}$ we denote the set $\left\{G^{I} \mid G \in \Gamma\right\} ;(\Gamma \Rightarrow F)^{I}$ stands for $\Gamma^{I} \Rightarrow F^{I}$.

Lemma 2

For any sequent $S$ and any interpretation $I$, if $S$ is a theorem of the basic system then so is $S^{I}$. 


\section{Proof}

Consider the property of sequents: " $S^{I}$ is a theorem of the basic system." To prove the lemma, it suffices to show that all theorems of the basic system have that property. It is clear that the reduct of every axiom of the basic system is a theorem (of the basic system). Verifying that the set of sequents with that property is closed under inference rules follows the same pattern for all inference rules but those involving implication. Consider, for instance, disjunction elimination:

$$
\frac{\Gamma \Rightarrow \mathcal{H}^{\vee} \quad \Delta, H \Rightarrow F \quad \text { for all } H \in \mathcal{H}}{\Gamma, \Delta \Rightarrow F}
$$

and assume that the reducts of all sequents that are premises of that rule are theorems. Because $\left(\mathcal{H}^{\vee}\right)^{I}$ is $\left(\mathcal{H}^{I}\right)^{\vee}$, all premises of the disjunction elimination rule:

$$
\frac{\Gamma^{I} \Rightarrow\left(\mathcal{H}^{I}\right)^{\vee} \quad \Delta^{I}, H^{I} \Rightarrow F^{I} \quad \text { for all } H \in \mathcal{H}}{\Gamma^{I}, \Delta^{I} \Rightarrow F^{I}}
$$

are theorems. Therefore, so is the sequent $\Gamma^{I}, \Delta^{I} \Rightarrow F^{I}$ and consequently, also the sequent $(\Gamma, \Delta \Rightarrow F)^{I}$.

Consider now the implication introduction rule:

$$
\frac{\Gamma, F \Rightarrow G}{\Gamma \Rightarrow F \rightarrow G}
$$

and assume that the reduct $(\Gamma, F \Rightarrow G)^{I}$ is a theorem. To show that $(\Gamma \Rightarrow F \rightarrow G)^{I}$ is a theorem it suffices to show that $\Gamma^{I} \Rightarrow(F \rightarrow G)^{I}$ is a theorem.

Case 1: $I$ satisfies $\Gamma$. Since the sequent $(\Gamma, F \Rightarrow G)^{I}$ is a theorem, so is the sequent $\Gamma^{I}, F^{I} \Rightarrow G^{I}$. Thus, $\Gamma^{I} \Rightarrow F^{I} \rightarrow G^{I}$ is a theorem and so, $\left(\Gamma^{I}\right)^{\wedge} \rightarrow\left(F^{I} \rightarrow G^{I}\right)$ is tautological. Since $I$ satisfies $\Gamma$, the comment at the end of Section 2 implies that $I$ satisfies $\Gamma^{I}$. Consequently, $I$ satisfies $F^{I} \rightarrow G^{I}$ and, by the same comment again, also $F \rightarrow G$. It follows that $(F \rightarrow G)^{I}$ is $F^{I} \rightarrow G^{I}$. Since the sequent $(\Gamma, F \Rightarrow G)^{I}$ or, equivalently, the sequent $\Gamma^{I}, F^{I} \Rightarrow G^{I}$ is a theorem, applying the rule

$$
\frac{\Gamma^{I}, F^{I} \Rightarrow G^{I}}{\Gamma^{I} \Rightarrow F^{I} \rightarrow G^{I}}
$$

we obtain that $\Gamma^{I} \Rightarrow F^{I} \rightarrow G^{I}$ is a theorem. Thus, $\Gamma^{I} \Rightarrow(F \rightarrow G)^{I}$, is a theorem, too.

Case 2: $I$ does not satisfy $\Gamma$. Then $I$ does not satisfy one of the elements $H$ of $\Gamma$. By Lemma $1, H^{I} \Rightarrow \perp$ is a theorem, and $\Gamma^{I} \Rightarrow(F \rightarrow G)^{I}$ can be derived from $H^{I} \Rightarrow \perp$ by rules $(C)$ and $(W)$. Thus, it is a theorem.

Next, consider the implication elimination rule:

$$
\frac{\Gamma \Rightarrow F \quad \Delta \Rightarrow F \rightarrow G}{\Gamma, \Delta \Rightarrow G}
$$

and assume that the sequents $(\Gamma \Rightarrow F)^{I}$ and $(\Delta \Rightarrow F \rightarrow G)^{I}$ are theorems. We will show that $(\Gamma, \Delta \Rightarrow G)^{I}$ or, equivalently, $\Gamma^{I}, \Delta^{I} \Rightarrow G^{I}$ is a theorem, too.

Case 1: $I$ satisfies $F \rightarrow G$. Then $(F \rightarrow G)^{I}$ is $F^{I} \rightarrow G^{I}$. Thus, the sequents 
$\Gamma^{I} \Rightarrow F^{I}$ and $\Delta^{I} \Rightarrow F^{I} \rightarrow G^{I}$ are theorems, and the claim follows by applying the rule

$$
\frac{\Gamma^{I} \Rightarrow F^{I} \quad \Delta^{I} \Rightarrow F^{I} \rightarrow G^{I}}{\Gamma^{I}, \Delta^{I} \Rightarrow G^{I}} .
$$

Case 2: $I$ does not satisfy $F \rightarrow G$. Then $(F \rightarrow G)^{I}$ is $\perp$ and so, $\Delta^{I} \Rightarrow \perp$ is a theorem. Moreover, $\Gamma^{I}, \Delta^{I} \Rightarrow G^{I}$ can be derived from $\Delta^{I} \Rightarrow \perp$ by rules $(C)$ and $(W)$. Thus, $\Gamma^{I}, \Delta^{I} \Rightarrow G^{I}$ is a theorem, too.

\section{Proof of Main Theorem}

(a) Assume that $F$ is a theorem of the basic system. By Lemma 2, for any interpretation $I, F^{I}$ is a theorem of the basic system, and consequently is tautological, by Proposition 2. It follows that $\mathcal{H}^{I}$ and $(\mathcal{H} \cup F)^{I}$ are satisfied by the same interpretations.

(b) Assume that $F$ is equivalent to $G$ in the basic system, that is, $F \leftrightarrow G$ is a theorem of the basic system. By Lemma 2, for every interpretation $I,(F \leftrightarrow G)^{I}$ is a theorem of the basic system. Moreover, by Proposition $2, F \leftrightarrow G$ is tautological. Thus $(F \leftrightarrow G)^{I}=F^{I} \leftrightarrow G^{I}$ and so, $F^{I} \leftrightarrow G^{I}$ is a theorem of the basic system. Consequently, $F^{I} \leftrightarrow G^{I}$ is tautological, that is $F^{I}$ and $G^{I}$ are equivalent. It follows that $(\mathcal{H} \cup F)^{I}$ and $(\mathcal{H} \cup G)^{I}$ are satisfied by the same interpretations.

\section{Examples Involving Aggregates}

As discussed in the introduction, infinitary formulas can be used to precisely define the semantics of aggregates in ASP when the Herbrand universe is infinite. In this section, we give two examples demonstrating how the theory described above can be applied to prove equivalences between programs involving aggregates.

Example 5

Intuitively, the rule

$$
q(X) \leftarrow 1\{p(X, Y)\}
$$

has the same meaning as the rule

$$
q(X) \leftarrow p(X, Y)
$$

To make this claim precise, consider first the result of grounding rule (18) under the assumption that the Herbrand universe $C$ is finite. In accordance with standard practice in ASP, we treat variable $X$ as global and $Y$ as local. Then the result of grounding (18) is the set of ground rules

$$
q(a) \leftarrow 1\{p(a, b) \mid b \in C\}
$$

for all $a \in C$. In the spirit of the semantics for aggregates proposed by Ferraris [2005, Section 4.1] these rules have the same meaning as the propositional formulas

$$
\left(\bigvee_{b \in C} p(a, b)\right) \rightarrow q(a)
$$


Likewise, rule (19) can be viewed as shorthand for the set of formulas

$$
p(a, b) \rightarrow q(a)
$$

for all $a, b \in C$. It easy to see that these sets of formulas are intuitionistically equivalent.

How can we lift the assumption that the Herbrand universe is finite? We can treat (20) as an infinitary formula, and show that the conjunction of formulas (20) is equivalent to the conjunction of formulas (21) in the basic system. The fact that the conjunction of formulas (21) for all $b \in C$ is equivalent to (20) in the basic system follows from Example 2 (Section 3).

\section{Example 6}

Intuitively,

$$
q(X) \leftarrow 2\{p(X, Y)\}
$$

has the same meaning as the rule

$$
q(X) \leftarrow p(X, Y 1), p(X, Y 2), \quad Y 1 \neq Y 2
$$

To make this claim precise, consider the infinitary formulas corresponding to (22):

$$
\left(\bigvee_{b \in C} p(a, b) \wedge \bigwedge_{b \in C}\left(p(a, b) \rightarrow \bigvee_{\substack{c \in C \\ c \neq b}} p(a, c)\right)\right) \rightarrow q(a)
$$

$(a \in C)$; see (Ferraris 2005, Section 4.1) for details on representing aggregates with propositional formulas. The formulas corresponding to (23) are

$$
(p(a, b) \wedge p(a, c)) \rightarrow q(a)
$$

$(a, b, c \in C, b \neq c)$. We will show that the conjunction of formulas (24) is equivalent to the conjunction of formulas (25) in the basic system.

It is sufficient to check that for every $a \in C,(24)$ is equivalent to the conjunction of formulas (25) over all $b, c \in C$ such that $b \neq c$. By Example 2, this conjunction is intuitionistically equivalent to

$$
\left(\bigvee_{\substack{b, c \in C \\ b \neq c}}(p(a, b) \wedge p(a, c))\right) \rightarrow q(a) .
$$

By the replacement property of infinitary formulas, it suffices to check that the antecedents of (24) and (26) are equivalent to each other.

Left-to-right: assume

$$
\bigvee_{b \in C} p(a, b) \wedge \bigwedge_{b \in C}\left(p(a, b) \rightarrow \bigvee_{\substack{c \in C \\ c \neq b}} p(a, c)\right)
$$


Then $\bigvee_{b \in C} p(a, b)$. We will reason by cases, with one case corresponding to each possible value $b_{0}$ of $b$. Case $p\left(a, b_{0}\right)$ : by the second conjunctive term of $(27)$,

$$
p\left(a, b_{0}\right) \rightarrow \bigvee_{\substack{c \in C \\ c \neq b_{0}}} p(a, c)
$$

Then the consequent of this implication follows. Again we will reason by cases, with one case for each value $c_{0}$ of $c$ where $c_{0} \neq b_{0}$. Case $p\left(a, c_{0}\right)$ : then $p\left(a, b_{0}\right) \wedge p\left(a, c_{0}\right)$. Consequently

$$
\bigvee_{\substack{b, c \in C \\ b \neq c}} p(a, b) \wedge p(a, c)
$$

Right-to-left: assume (28). We reason by cases, with one case for each pair $b_{0}, c_{0}$, where $b_{0} \neq c_{0}$. Case $p\left(a, b_{0}\right) \wedge p\left(a, c_{0}\right)$ : from $p\left(a, b_{0}\right)$ we derive the first conjunctive term of $(27)$; from $p\left(a, c_{0}\right)$ we derive

$$
\bigvee_{\substack{c \in C \\ c \neq b}} p(a, c)
$$

and consequently the implication

$$
p(a, b) \rightarrow \bigvee_{\substack{c \in C \\ c \neq b}} p(a, c) .
$$

The conjunction of these implications for all $b \in C$ is the second conjunctive term of (27).

\section{The Extended System of Natural Deduction}

In this section, we show that the assertion of the main theorem will remain true if we extend the basic system by adding the axiom schema

$$
F \vee(F \rightarrow G) \vee \neg G
$$

characterizing (in the finite case) the logic of here-and-there (Hosoi 1966), and the converses to the implications discussed at the end of Section 3:

$$
\begin{aligned}
& \neg \bigwedge_{F \in \mathcal{H}} F \rightarrow \bigvee_{F \in \mathcal{H}} \neg F, \\
& \left(\bigwedge_{i \in I} \bigvee_{F \in \mathcal{H}_{i}} F\right) \rightarrow\left(\bigvee_{\left\{F_{i}\right\}_{i \in I}} \bigwedge_{i \in I} F_{i}\right),
\end{aligned}
$$

and

$$
\left(\bigwedge_{\left\{F_{i}\right\}_{i \in I}} \bigvee_{i \in I} F_{i}\right) \rightarrow\left(\bigvee_{i \in I} \bigwedge_{F \in \mathcal{H}_{i}} F\right)
$$

When all conjunctions and disjunctions are finite, formula (30) can be derived intuitionistically from (29), and (31) and (32) are intuitionistically provable. We 
do not know to what extent the additional axiom schemas postulated here are independent when infinite conjunctions and disjunctions are allowed.

In the extended system, we can derive the theorem

$$
\bigvee_{J \subseteq I}\left(\neg \bigvee_{j \in I \backslash J} F_{j} \wedge \neg \neg \bigwedge_{j \in J} F_{j}\right)
$$

for any non-empty bounded family $\left\{F_{i}\right\}_{i \in I}$ of formulas. (This is a generalization of the weak law of the excluded middle $\neg F \vee \neg \neg F$ to sets of infinitary formulas, similar to the generalization of the law of the excluded middle given in Remark 2. It is equivalent in the basic system to the special case of (33) corresponding to a family with a single element.) Indeed

$$
\bigwedge_{i \in I}\left(\neg F_{i} \vee \neg \neg F_{i}\right)
$$

is a theorem of the extended system because $\neg F_{i} \wedge \neg \neg F_{i}$ can be intuitionistically derived from (29) with $F_{i}$ as $F$ and $\neg F_{i}$ as $G$. Using (31) we obtain

$$
\bigvee_{J \subseteq I}\left(\bigwedge_{j \in I \backslash J} \neg F_{j} \wedge \bigwedge_{j \in J} \neg \neg F_{j}\right),
$$

and (33) follows by De Morgan's laws.

In the extended system, we can also derive the theorem

$$
\left(F \rightarrow \bigvee_{i \in I} G_{i}\right) \rightarrow \bigvee_{i \in I}\left(F \rightarrow G_{i}\right)
$$

for any formula $F$ and non-empty family $\left\{G_{i}\right\}_{i \in I}$ of formulas. We use instantiations of (29) for all $G_{i}$ to obtain

$$
\bigwedge_{i \in I} F \vee\left(F \rightarrow G_{i}\right) \vee \neg G_{i}
$$

By (31) we obtain

$$
\bigvee_{\left\{F_{i}\right\}_{i \in I}} \bigwedge_{i \in I} F_{i}
$$

where the disjunction extends over all elements $\left\{F_{i}\right\}_{i \in I}$ of the Cartesian product of the family $\left\{F, F \rightarrow G_{i}, \neg G_{i}\right\}_{i \in I}$. We reason by cases, with one case corresponding to each disjunctive term $\bigwedge_{i \in I} F_{i}$ of (35). If at least one of the formulas $F_{i}$ is $F$ then from the antecedent of (34) we can derive $\bigvee_{i \in I} G_{i}$, and the consequent of (34) immediately follows. If at least one of the formulas $F_{i}$ is $F \rightarrow G_{i}$ then the consequent of (34) is immediate as well. Otherwise, $\bigwedge_{i \in I} F_{i}$ is $\bigwedge_{i \in I} \neg G_{i}$. Then from the antecedent of (34) we can derive $\neg F$ and every disjunctive term of the consequent follows.

It is easy to check that the properties of the basic system proved in Section 4 hold for the extended system as well. 
To show that the assertion of the main theorem applies to the extended system we will prove the modification of Lemma 2 stated below. The classical extended system is obtained from the extended system by replacing the axiom schema (29) with the law of the excluded middle (9).

Lemma 3

For any sequent $S$ and any interpretation $I$, if $S$ is a theorem of the extended system then $S^{I}$ is a theorem of the classical extended system.

Proof

It suffices to show that every theorem $S$ of the extended has this property: " $S$ " is a theorem of the classical extended system." We only need to check that the reducts of the axioms (29)-(32) have this property; the fact that the set of sequents with that property is closed under the inference rules is checked in the same way as in the proof of Lemma 2.

Let $S$ be (29). Then $S^{I}$ is

$$
F^{I} \vee(F \rightarrow G)^{I} \vee(\neg G)^{I}
$$

If $I \models G$ then the second disjunctive term is $F^{I} \rightarrow G^{I}$, and the disjunction can be derived from $F^{I} \vee \neg F^{I}$. If $I \forall \forall G$ then the third disjunctive term is equivalent to $\neg \perp$.

Let $S$ be (30). Since $S$ is tautological, $S^{I}$ is

$$
\left(\neg \bigwedge_{F \in \mathcal{H}} F\right)^{I} \rightarrow \bigvee_{F \in \mathcal{H}}(\neg F)^{I} .
$$

If $I$ satisfies the conjunction in the antecedent, then the antecedent is $\perp$. Otherwise, at least one disjunctive term in the consequent is equivalent to $\neg \perp$.

Let $S$ be (31). Since $S$ is tautological, $S^{I}$ is

$$
\left(\bigwedge_{i \in I} \bigvee_{F \in \mathcal{H}_{i}} F^{I}\right) \rightarrow\left(\bigvee_{\left\{F_{i}\right\}_{i \in I}} \bigwedge_{i \in I} F_{i}^{I}\right)
$$

which is an instantiation of the same axiom schema. The reasoning for sequents of the form (32) is similar.

Main Theorem for the Extended System

For any set $\mathcal{H}$ of formulas,

(a) if a formula $F$ is a theorem of the extended system then $\mathcal{H} \cup\{F\}$ has the same stable models as $\mathcal{H}$;

(b) if $F$ is equivalent to $G$ in the extended system then $\mathcal{H} \cup\{F\}$ and $\mathcal{H} \cup\{G\}$ have the same stable models.

This assertion is derived from Lemma 3 in the same way that the Main Theorem was derived from Lemma 2, using the fact that all theorems of the classical extended system are tautological. 


\section{Example 7}

Intuitively, the cardinality constraint $\{p(X)\} 0$ ("the set of true atoms with form $p(X)$ has cardinality at most 0 ") has the same meaning as the conditional literal $\perp: p(X)$ ("for all $X, p(X)$ is false"). If we represent this conditional literal by the infinitary formula

$$
\bigwedge_{a \in C} \neg p(a)
$$

then this claim can be made precise by showing that (36) is equivalent in the extended system to the infinitary formula corresponding to $\{p(X)\} 0$ in the sense of (Ferraris 2005):

$$
\bigwedge_{\substack{A \subseteq C \\ A \neq \emptyset}}\left(\bigwedge_{a \in A} p(a) \rightarrow \bigvee_{a \in C \backslash A} p(a)\right)
$$

(where $C$ is the Herbrand universe).

It is easy to derive (37) from (36) in the basic system. The derivation of (36) from (37) will use the following instance of (33):

$$
\bigvee_{A \subseteq C}\left(\neg \bigvee_{a \in C \backslash A} p(a) \wedge \neg \neg \bigwedge_{a \in A} p(a)\right) .
$$

We will reason by cases, with one case corresponding to each disjunctive term $D_{A}$ in (38). In the case that $A$ is empty, (36) follows from the first conjunctive term of $D_{A}$ by De Morgan's law. Otherwise, assume $\bigwedge_{a \in A} p(a)$. Then by (37), $\bigvee_{a \in C \backslash A} p(a)$, which contradicts the first conjunctive term of $D_{A}$. We conclude $\neg \bigwedge_{a \in A} p(a)$, which contradicts the second conjunctive term of $D_{A}$. So the assumptions $D_{A}$ and (37) are contradictory. Consequently, they imply (36).

\section{Conclusion}

Two finite propositional formulas are strongly equivalent if and only if they are equivalent in the logic of here-and-there (Ferraris 2005, Proposition 2). The results of this note are similar to the if part of that theorem. We don't know how to extend the only if part to infinitary formulas. It is not clear whether or not any axioms or inference rules not included in the extended system will be required. However, as we illustrated with several examples, the results in this paper allow us to verify the equivalence of formulas involving aggregates.

\section{Acknowledgements}

Thanks to Fangkai Yang and to the anonymous referees for comments. 


\section{References}

Ferraris, P. 2005. Answer sets for propositional theories. In Proceedings of International Conference on Logic Programming and Nonmonotonic Reasoning (LPNMR). 119-131.

Gelfond, M. And Lifschitz, V. 1988. The stable model semantics for logic programming. In Proceedings of International Logic Programming Conference and Symposium, R. Kowalski and K. Bowen, Eds. MIT Press, 1070-1080.

HARrison, A. 2013. On the semantics of Gringo and proving strong equivalence. In Theory and Practice of Logic Programming, Online Supplement.

Harrison, A., Lifschitz, V., And Truszczynski, M. 2013. On equivalent transformations of infinitary formulas under the stable model semantics (preliminary report). In Proceedings of International Conference on Logic Programming and Nonmonotonic Reasoning (LPNMR). 387-394.

Hosor, T. 1966. The axiomatization of the intermediate propositional systems $S_{n}$ of Gödel. Journal of the Faculty of Science of the University of Tokyo 13, 183-187.

Kalmár, L. 1935. Über die Axiomatisierbarkeit des Aussagenkalküls. Acta Sci. Math. 7, $222-243$.

KARP, C. R. 1964. Languages with expressions of infinite length. North-Holland, Amsterdam.

Lifschitz, V., Morgenstern, L., And Plaisted, D. 2008. Knowledge representation and classical logic. In Handbook of Knowledge Representation, F. van Harmelen, V. Lifschitz, and B. Porter, Eds. Elsevier, 3-88.

Lifschitz, V., Pearce, D., And Valverde, A. 2001. Strongly equivalent logic programs. ACM Transactions on Computational Logic 2, 526-541.

LifsChitz, V. AND YANG, F. 2012. Lloyd-Topor completion and general stable models. In Working Notes of the 5th Workshop of Answer Set Programming and Other Computing Paradigms (ASPOCP 2012).

Pearce, D. 1997. A new logical characterization of stable models and answer sets. In NonMonotonic Extensions of Logic Programming (Lecture Notes in Artificial Intelligence 1216), J. Dix, L. Pereira, and T. Przymusinski, Eds. Springer, 57-70.

ScOTt, D. AND TARSKI, A. 1958. The sentential calculus with infinitely long expressions. In Colloquium Mathematicae. Vol. 6. 165-170.

Truszczynski, M. 2012. Connecting first-order ASP and the logic FO(ID) through reducts. In Correct Reasoning: Essays on Logic-Based AI in Honor of Vladimir Lifschitz. Springer. 